\title{
Autopilot: A Paradigm Revolution In The Field Of Road Traffic And A New Engine For The Construction Of A Traffic Power
}

\author{
GengRui
}

Reseach Institute of Highway Ministry of Transport, Beijing, china

\begin{abstract}
Keywords: Transportation engineering; Transport economics; Strategy.
Abstract: Introduces the fundamental changes caused by autopilot to road facilities, transportation services and management methods. Proves the key problem of whether we can build a powerful traffic power in the field of highway traffic is to whether the transformation can be successfully changed from the traditional car paradigm to the autopilot. Puts forward some suggestions on how to carry out the transformation of the road transport industry smoothly.
\end{abstract}

Paradigm concept is the scientific philosopher Thomas Kuhn in "the structure of scientific revolutions" (The Structure of Scientific Revolution) in the field, is a common acceptance of a hypothesis, theory, methods and beliefs and the total. For the general development of science and technology, the so-called technological revolution is not simply a scientific change from quantitative change to qualitative change, but a paradigm shift. That is, only a fundamental transformation in basic hypotheses, scientific methods and common beliefs will lead to scientific revolution. If continuous improvement is a quantitative change from 1 to 100 , then the paradigm shift is a breakthrough of 0 to 1 .

\section{Autopilot is a paradigm revolution in the field of road traffic}

The development stage of our road traffic is the paradigm shift from "carriage" to "car". More than 2000 years ago, the first emperor of Qin began to carry out the "car track". At that time, the "car" on the same track was the coach, and the track was the two hard road with the same wheel width after rolling repeatedly on the soil road. Traffic has experienced more than 2000 years of evolution leading to the carriage, 1885 Carle Benz invented the world first car as a symbol of human traffic has gradually entered the "auto" paradigm, from the paradigm of about $25 \mathrm{~km} /$ hour speed of about $100 \mathrm{~km} /$ hour to leap about speed.

In 1901, the first vehicle in China was shipped to Shanghai. By 1956, the first Jiefang car was produced, and then the car explosion entered the family in the 90s of last century. Until now, road traffic is developing in the "automobile" paradigm. In the paradigm of human driving, the corresponding road system, vehicle rear service system, road transport service system and road traffic management system are gradually constructed. Now the car has been the first round into a four round, from $30 \mathrm{~km} / \mathrm{h}$ to $100 \mathrm{~km} /$ hour, from manual to automatic, driven by fuel into electricity, configuration upgrade, performance improvement, but they are in the "car" paradigm are improved by 1 to 100 , in the fast at the same time the development of accumulated safety, pollution, congestion and other thorny issues, people are trying to solve, but the effect is not ideal.

Einstein once pointed out that the major problems we face can never be solved at the level of the problem itself. For example, the horse dung disposal is always the biggest problem for the public and managers in the carriage mode, and the problem is also gone with the wind. In the same way, 
the social problems such as congestion and pollution caused by the automobile paradigm are difficult to cure under the auto paradigm of the problem itself. With the development of artificial intelligence breakthrough, make "autopilot" possible, it will bring about fundamental changes in terms of personnel, road facilities, transportation services, governance, social problems in the automobile paradigm will also collapse of itself in the autopilot paradigm, the autopilot will become the road traffic development paradigm shift direction.

(1) traffic efficiency will be greatly improved.

The autopilot is on the radar image recognition, automatic identification technology as the foundation, to ensure the safety of the premise to realize the automatic fast driving mode, and car networking technology to ensure the communication speed before and after the car, hundreds of cars can be compared before and after the close, and depend on people's attention, reaction speed and operation level is the bottleneck of the ceiling the traditional car, artificial intelligence and human in terms of efficiency magnitude difference.

(2) the requirements of the automatic driving vehicle to the road system will change radically.

The autopilot can overcome the centrifugal force by technical means more flexible function, also can drive to 120 yards per hour in the four plane linear road safety, which will overturn the "people's perception based road design theory, automatic driving on road alignment requirements will be reduced, the total size of the supply and demand of road will be reduced, but on the road to provide information perception and self perception of vehicle maintenance will put forward higher requirements.

(3) autopilot will change the mode of transport service supply.

Not only small cars, buses, long-distance passenger and cargo vehicles can be automatic driving, transportation service supply mode will have a major change. Traditional transport enterprises will be replaced by technology companies and car companies that offer travel solutions. Automatic driving is a natural upgrading of Uber and drip services, but no need for human driving. The time sharing rental service will replace the existing large-scale private purchase cars. For the user, the car is no longer a commodity but only a service. It does not need to be used only. Autopilot will provide a 24 hour door to door reservation service without the need to consider the driver's 8 hour working system and prevent fatigue driving.

(4) autopilot will make the social problems such as congestion and pollution break out (no cure), and travel will be a part of a good life.

Under the traditional automobile paradigm, traffic jam and environmental pollution have become an incurable disease of the city. Modern people have to endure the time and air pollution on the road every day. But due to the sudden increase of electric drive and efficiency, automatic driving will not only become invisible, such as congestion and pollution, but also liberate people's attention and continue to work on cars, or enjoy various kinds of entertainment.

(5) autopilot will also completely change the traffic control structure and management system.

Automatic driving will change all the current driving safety management system, to increase the safety of the vehicle technology management, increase information collection, to ensure the safety of vehicles traveling exchange, data security management, also need to re design of the automatic driving accident "responsibility" and the traffic insurance system, all laws and regulations the system will, because from the "people" to drive "car" driving and the need to re design and regulation. 


\section{To build a powerful traffic, it is necessary to take the lead in realizing the paradigm shift of autopilot}

Since 2010, when Google began to test its automatic driving vehicle on public roads, many industrial power including traditional car companies, Internet giants and start-ups joined in. Not only has Tesla, Google, Uber and other scientific and technological enterprises, but also Ford, BMW, TOYOTA and other traditional car enterprises also joined the technology competition. From the overall progress of the situation, self driving cars still remain in the stage of basic technology research and development, in the third stage of automatic driving -- "conditional automatic driving" stage, did not reach the fifth stage: "fully automatic driving" stage, has not yet entered commercial production stage, but did not enter the substantive operation of transport field service.

Since 2010, when Google began to test its automatic driving vehicle on public roads, many industrial power including traditional car companies, Internet giants and start-ups joined in. Not only has Tesla, Google, Uber and other scientific and technological enterprises, but also Ford, BMW, TOYOTA and other traditional car enterprises also joined the technology competition. From the overall progress of the situation, self driving cars still remain in the stage of basic technology research and development, in the third stage of automatic driving -- "conditional automatic driving" stage, did not reach the fifth stage: "fully automatic driving" stage, has not yet entered commercial production stage, but did not enter the substantive operation of transport field service.

In 2017, the nineteen major reports of the party put forward the goal of the middle of the century, and put forward the strategic task of building a powerful traffic power. This 30 years, road traffic also coincided with the paradigm shift change, opportunity, can smoothly from the traditional paradigm shift to the automatic driving car paradigm, will determine whether we build traffic power key problems in the field of highway traffic, because the new paradigm of the old paradigm is the comparative advantages of rolling type number level and the. If all countries are in the new paradigm, China will not always be the best, but if other countries have replaced the new paradigm, we are also immersed in the old paradigm to improve and improve, so it is totally uncompetitive.

The connotation of traffic power, one is reflected in the traffic of passenger and freight transport service object better in security, efficiency and convenience etc.; the two is reflected in the domestic and international comparison, leading technology, novel model, standard, advanced discourse and influential and dominant etc.

For the first connotation, automatic driving and traditional car rolling advantages in safety, efficiency and convenience are not necessary. For the second China's connotation, in the traditional automotive paradigm, due to the closed, war and other reasons, started later than the western developed countries for nearly 70 years, so whether it is the technical standard of road transport vehicles, technology and management technology can only be used as a follower, introduction and absorption of western mature technology, although China now no road the scale of infrastructure, construction technology, etc. the total transport are located in the forefront of the world, but the underlying theory and techniques are based on foreign, China's only large-scale users, rather than the original, and then a large scale can only be called traffic power, no energy is called power. Now, in the face of road traffic pattern automatic driving will bring the trend, China and other developed countries almost at the same starting line, if the first and the smooth realization of the paradigm shift in the field of transportation, the large-scale application of automatic driving, road traffic will have innovative development opportunities, provide China model will be in the relevant system, the standard and the management pattern, then in the world of traffic discourse, influence and leadership will be self-evident. 


\section{Strategic layout for autopilot}

In order to meet the arrival of the autopilot paradigm, it is suggested that the transportation industry start from the following three aspects

One is to study and construct a road coordination system adapted to automatic driving. The technology development of automatic driving is mainly led by technology enterprises and automobile manufacturers. Providing an adaptive road system is an important responsibility of the transportation industry. We should actively connect the new requirements of the automatic driving technology to the road system, and actively research and build a road, technology system, construction system and operation system that adapts, assists or voluntarily guides the automatic driving vehicle.

The two is to encourage innovation based transportation service supply model based on automatic driving. Self driving cars will give users tend to not only pay the purchase of vehicles, automatic driving to provide travel services, science and technology enterprises and large enterprises will become the main road transport service, they will build a new scene based on replacing the traditional mass transit service supply to instant service mode of large scale private custom, encourage service mode innovation, implementation of bottom line supervision, "let the bullets fly for a while, the government of the autonomous vehicle rapid popularization of the best policy support.

The three is to build a new framework for the management system for automatic driving. Autopilot makes the focus of management change from "driver" to "vehicle" and "information". The driver's related management system will gradually withdraw, and we need to rebuild a new governance structure based on automatic driving vehicle manufacturing, using, technology updating and data collaboration. During the period, the excessive phase of autopilot and traditional car mixing is also an insurmountable and more intelligent governance stage.

Baigezhengliu, fall behind, slow retreat! In July 27, 2017, the United States House of Representatives unanimously passed the bipartisan act Self Drive Act. It will manage for the first time the production, testing and release of the automatic driving vehicle. This bill will be the first federal law to accelerate the listing of automatic driving vehicles in the United States, which has a policy of "exemption from supervision" to a certain number of self driving cars manufactured by the automakers in the first 4 years.

In the face of new opportunities for automatic driving traffic transfer paradigm brings, in other countries the government fully supports the automatic driving of new initiatives, in the face of the autopilot is going from the point to the new technology research and development and production, to enter the field of transport services, China should seize the opportunity in the field of road traffic flow, and strive to achieve the transfer to the automatic driving mode the power to seize the opportunities for the construction of traffic.

\section{References}

[1]Yang Peikun Intelligent transportation, compiling Tongji University press, 2016.7

[2]Dayi intelligent transportation technology and its application, edited by Wei Jinli Mechanical Industry Press, 2016

[3]Introduction of intelligent transportation system engineering, editor of Electronic Industry Press, editor in chief of Zhang Guowu, 2015 